\title{
Geometries and Electronic States of Divacancy Defect in Finite-Size Hexagonal Graphene Flakes
}

\author{
Lili Liu ${ }^{1}$ and Shimou Chen ${ }^{2}$ \\ ${ }^{1}$ Department of Chemistry, School of Science, Beijing Technology and Business University, Beijing 100048, China \\ ${ }^{2}$ Key Laboratory of Green Process and Engineering, Institute of Process Engineering, Chinese Academy of Sciences, \\ Beijing 100190, China \\ Correspondence should be addressed to Shimou Chen; chenshimou@ipe.ac.cn
}

Received 14 December 2016; Accepted 4 January 2017; Published 29 January 2017

Academic Editor: Gang Feng

Copyright ( 12017 Lili Liu and Shimou Chen. This is an open access article distributed under the Creative Commons Attribution License, which permits unrestricted use, distribution, and reproduction in any medium, provided the original work is properly cited.

\begin{abstract}
The geometries and electronic properties of divacancies with two kinds of structures were investigated by the first-principles (U) B3LYP/STO-3G and self-consistent-charge density-functional tight-binding (SCC-DFTB) method. Different from the reported understanding of these properties of divacancy in graphene and carbon nanotubes, it was found that the ground state of the divacancy with 585 configurations is closed shell singlet state and much more stable than the 555777 configurations in the smaller graphene flakes, which is preferred to triplet state. But when the sizes of the graphene become larger, the 555777 defects will be more stable. In addition, the spin density properties of the both configurations are studied in this paper.
\end{abstract}

\section{Introduction}

Graphene, a single carbon plane arranged on a honeycomb lattice, has attracted immense investigation since its discovery in 2004 [1]. Lattice imperfections are introduced into graphene unavoidably during graphene growth or when irradiating a graphene sheet using high-energy particles [2-5]. These structural defects are known to significantly affect electronic and chemical properties [6,7]. In particular, the presence of defects can dramatically change the charge transport and magnetic properties of graphene due to disorder and localization effects, which are of important for their applications in molecular electronics. A comprehensive understanding of graphene defects is thus of critical importance.

Divacancy defect in graphene can be obtained either by the coalescence of two monovacancies or by removing two neighboring atoms. Although monovacancies had been widely studied [8-12], the divacancy as well as more larger vacancies could be frequently created in a physical or chemical treatment of graphene. For example, transmission electron microscopy experiments indicated that multivacancies, rather than monovacancies, more easily occur under moderate irradiation conditions [13]. Theoretical calculations at different levels of theory also point out that formation energies of divacancies in carbon nanotubes and graphene are much lower than monovacancy formation energies [14-18].

Typically, no dangling bond is present in a fully reconstructed divacancy so that two pentagons and one octagon (585 defects) appear, but the 585 defects are not the only possible way for a graphene lattice to accommodate two missing atoms [19]. Lee et al. found that the rotation of one of the bonds in the octagon of the 585 defects transforms the defect into an arrangement of three pentagons and three heptagons (555777 defects) [16, 20], which is also observed by the experiment study [21]. Even though high-resolution transmission electron microprobe methods enable an in situ measurement on the stability and migration of divacancy at atomic scale, the microscopic identification of the defects can not be an insight into the electronic properties of the defected graphene. In addition, as the edge (zigzag or armchair) plays a key role in the electronic and magnetic properties of graphene, as far as we know, there is still no report on investigating the influence on the electronic state of divacancy in graphene by edge effect. 
In this work, the geometries and electronic states of two preferable types of divacancy defects in finite-size hexagonal graphene flakes (HGFs) were calculated by selfconsistent-charge density-functional tight-binding (SCCDFTB) method for the first time, which are three pentagons and three heptagons composed (555777) divacancy defect structure and two pentagons side with octagon composed (585) structures, respectively. The formation energy, electronic state, and spin density distribution of different isomers were compared, and the edge effects on the properties of these different type divacancies were also studied.

\section{Methodology and Model Structures}

To calculate the geometries and electronic states of divacancy in graphene, we employed the computationally more economic self-consistent-charge density-functional tightbinding (SCC-DFTB) method in addition to first-principles (U) B3LYP/STO-3G level of theory to do the geometry optimization and the different spin states calculations. The SCCDFTB calculations were carried out with DFTB+, a program package developed by Aradi, Hourahine, and Frauenheim, implementing the original DFTB algorithms using sparsematrix techniques [22-26]. The electronic temperature $T_{\mathrm{e}}$ for the open shell state calculation was chosen to be $1000 \mathrm{~K}$, and the spin-polarized version of DFTB was employed for the high spin state calculation. For numerical vibrational frequencies calculation, we used GAUSSIAN 03 package with "external" keyword, calling SCC-DFTB $T_{\mathrm{e}}=1000 \mathrm{~K}$ external = "SCCDFTB" [27].

In this work, two series of typical divacancy defects in different sizes of zigzag- and armchair-edges hexagonal graphene flakes were investigated (Figure 1). One structure is three pentagons and three heptagons composed structures (555777) divacancy defect (highlighted by black color in Figure 1(a)), and the other type is two pentagons' side with an octagon composed (585) configuration (highlighted by black color in Figure 1(b)). There are different sizes of the flakes with divacancy for the same defect structure: from Z1 to Z12 for zigzag HGFs and from A1 to A6 for armchair HGFs (the nomination was shown in Figure 1). In addition, the small examples of the two defects are shown in Figures 1(b) and 1(c); we call these two types defects 585 and 555777, respectively. All these structures are minima, which have performed numerical vibrational frequencies calculation.

\section{Results and Discussion}

3.1. The Electronic State Properties of the Different Divacancy Isomers. In order to study the electronic characteristic of the divacancy defects, the energy differences between the different electronic states have been calculated on the two types of defects using both B3LYP/STO-3G and DFTB method and the results are shown in Figure 2. Figure 2(a) shows the energy differences of the same isomers with the different edge shape using B3LYP method, and Figure 2(b) shows the results using DFTB method; because the B3LYP is a very expensive calculation method for big size system, we only performed the finite-sized graphene flake. Both results of the energy differences with the two methods can be derived that DFTB method produced the same tendency of the energy differences with B3LYP method, and it is reliable and efficient in the really large carbon system.

Based on the comprehensive results of DFTB calculation in Figure 2, all the $\Delta E$ values $\left(\Delta E=E_{\text {triplet }}-E_{\text {singlet }}\right)$ of 555777 defects are negative except for $\mathrm{Z1}$ in zigzag-edge graphene flakes and all the values of 585 defects are positive except for Z11 and Z12. It stands for the notion that for the 555777 structures the ground states are more preferably triplet, while the 585 structures are preferred to be closed shell singlet within the zigzag HGFs. But for the divacancy in armchair HGFs, the energy difference curve is more flat, especially for the 555777 defects, the energies of singlet and triplet structures are very close to each other, all the ground states of 585 structures are closed shell states, and the 555777 defects are triplet states.

3.2. The Stability of 55577 and 585 Divacancies. The stability of the two different configurations of the divacancies in the same size and edge graphene also has been calculated with both DFT and DFTB methods. The results are shown in Figure 3. From the figure, it is easy to see that, for the medium sizes HGFs, the divacancy energy differences of the two levels of theories are very similar, which also proved that the DFTB is reliable for such system.

In addition, when the $\Delta E$ values, the energy differences between ground state energies of 585 defects (singlet state) and 555777 defects (triplet state), are positive, it means the 555777 is more stable, while when the value is negative, it means the 585 structures are more stable. From Figure 3, one can conclude that the stability has a very close relationship with both the HGFs edge shape and the defect structure. In armchair-edge HGFs, when the flakes size is smaller than A5 (A5 means the structure with a $18.5 \AA$ distance between the centers and the hexagon corner), the $\Delta E$ values are negative, and the 585 structure defects will be more stable than 555777 defects. That is to say, from A5 to bigger HGFs, the openshelled triplet 555777 divacancy defects changed to be more stable. But for zigzag-edge HGFs, this stability changed from Z8 (Z8 means the structure with a $21.0 \AA$ distance between the center and the hexagon corner), and from that structure, the 585 divacancies changed to be more stable than 555777 structures. So the stability of these two popular divacancies are highly depended on the structures themselves and also determined by the HGFs edges.

3.3. The Formation Energies of the Two Divacancy Isomers. In the former theoretical studies of the divacancy on graphene, it is reported that the 585 defects are less stable than 555777 defects about $0.8 \mathrm{eV}$ in graphene $[16,17]$. But in the nanotubes, it had been predicted that the formation energies for divacancies in armchair nanotubes are higher than in zigzag nanotubes. In addition, for the 585 divacancies in CNTs, the formation energies of divacancies strongly depend on the orientation of the divacancy with respect to the tube axis [27]. Lee et al. carried out the first-principles calculations and 


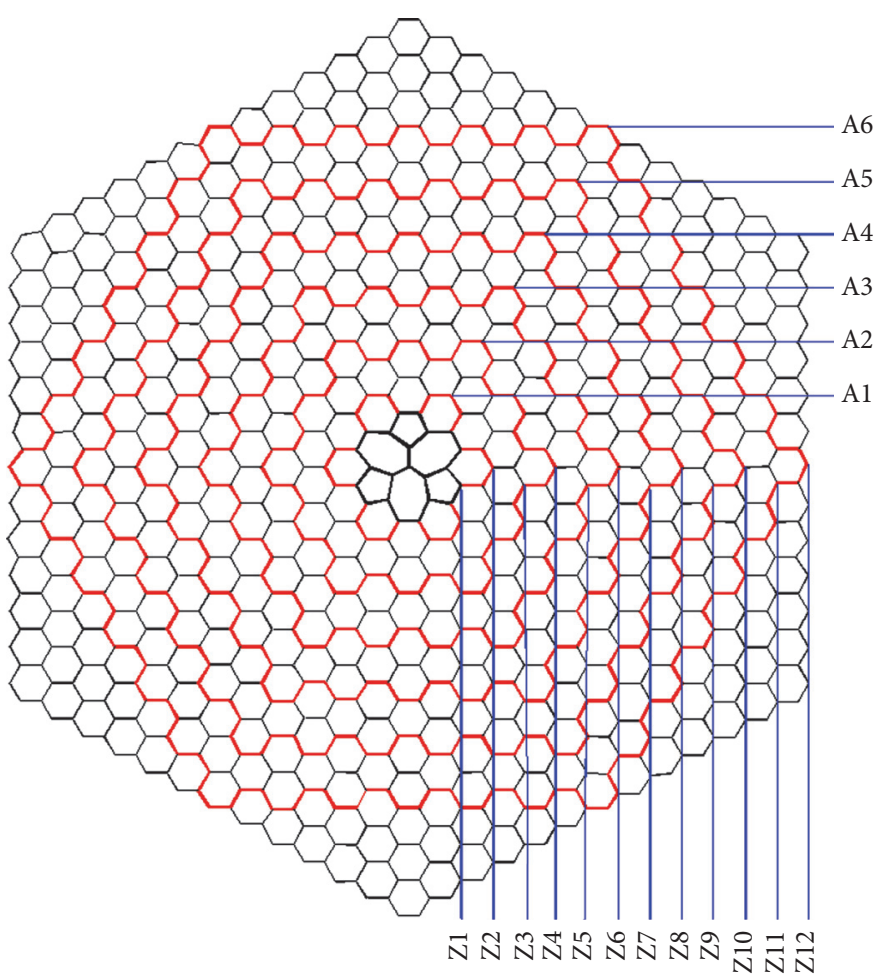

(a)

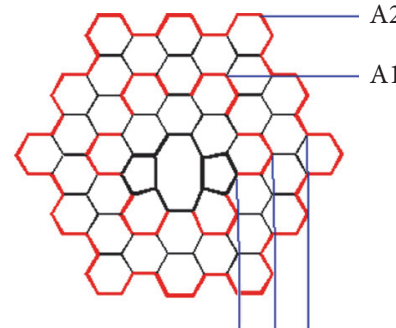

$\vec{N} \mathfrak{N}$

(b)

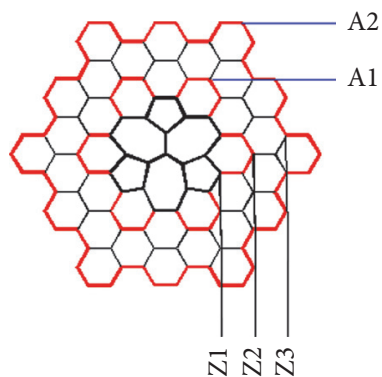

(c)

Figure 1: (a) The model structures of the two types divacancies, black bond stands for the zigzag-edge hexagonal graphene flakes and red bonds stand for the armchair ones. (b) Example for the small sizes of the zigzag (Z1, Z2, Z3) and armchair (A1, A2) hexagonal flakes with 585 defects in the center. (c) Example for the small sizes of the zigzag and armchair hexagonal flakes with 555777 defects in the center.

confirmed that the 555-777 defect is energetically much more stable in graphene [16]. In order to clarify the controversy result on this issue, here, we performed DFTB calculation on the formation energy of divacancy in HGFs with different size and edge of graphene.

The formation energy was calculated based on the following formula:

$$
\Delta E=E\left(\mathrm{Z}_{n}\right)-E\left(\mathrm{Z}_{n+2}\right)+2 * E(\text { C atom }),
$$

where the symbol $Z_{n}$ stands for the divacancy defects shown in Figure $1, Z_{n+2}$ stands for the HGFs without defects, and $Z$ represents the zigzag-edged graphene flakes. The armchair divacancy defects are calculated in the same way.

The results of our calculations are shown in Figure 4. The divacancy formation energies of the two isomers (555777 and 585) in the same HGFs are increasing along with the
HGFs size increase. For the zigzag-edge graphene flakes, the formation energy of the 555777 defects are higher than 585 defects in the size rang from $Z 1$ to $Z 7$, wherein, when the size of HGFs is equal to or bigger than Z8, the formation energy of 585 become more higher, which have the same tendency with the energy differences between these two isomers in the same flakes as shown in Figure 2. For the armchair-edge HGFs, the result of our calculation also shows the similar tendency on the formation energy of the two isomers, but, in this case, the turning point is A5, the formation energy of 555777 is higher than that of 585 when the size is smaller than A5 and then becomes lower when the size of armchair-edge graphene is the same or higher. Therefore, we obtained a general rule on the formation energy of graphene flakes, and we found that the absolute value of the formation energy has the same tendency with the energy differences between the two 

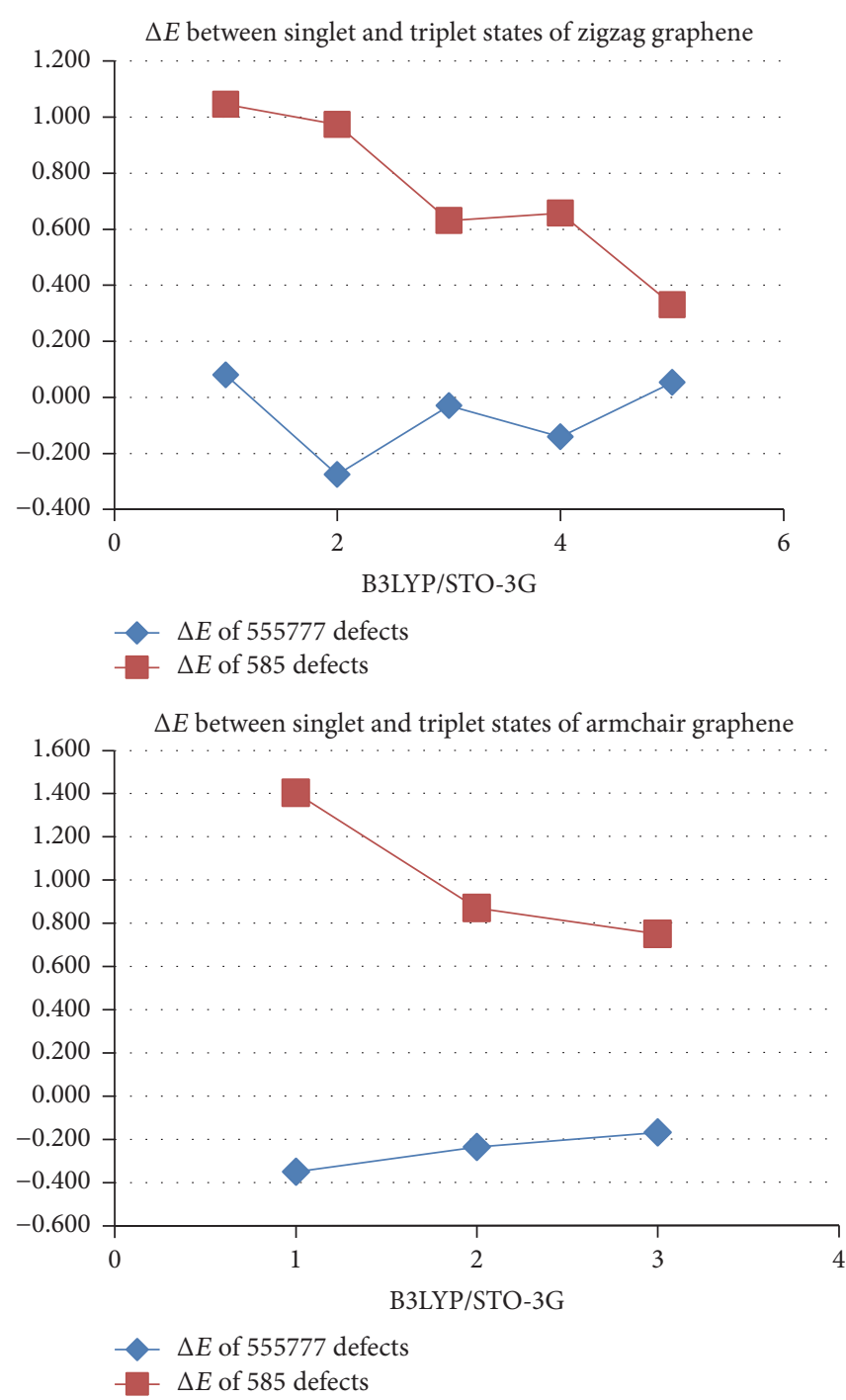

(a)
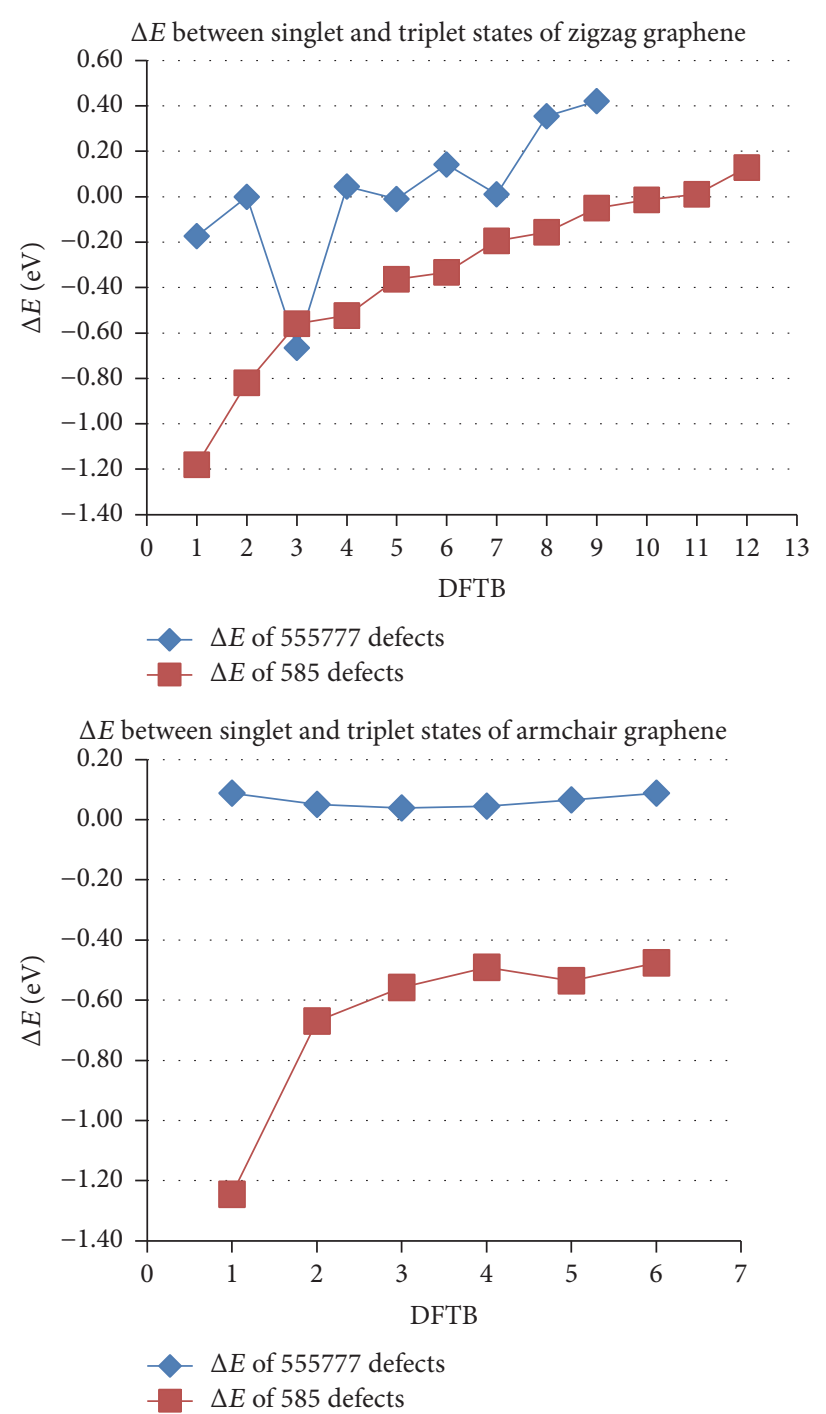

(b)

FIGURE 2: The energy differences between the singlet and triplet states of different types of divacancy defects with B3LYP/STO-3G and SDFTB methods, respectively.

isomers in the same flakes, and the stability of the defective flakes is highly dependent on the size of the graphene flakes and the defect structures.

\subsection{Spin Density Distribution of the Open-Shelled 555777} Defects. According to the analysis results above, the ground states of the two divacancy defects are totally different. The most stable electronic state of the 585 divacancies is close shell singlet without any unpaired electron, but for the 555777 structures, the energetically stable state is open shell triplet state, which has two unpaired electrons. The two unpaired electrons are evenly localized on three carbons that are directly connected to the center carbon atom and have been shared by both the pentagon and the heptagon. Some middle size zigzag and armchair 555777 divacancy graphene defects of the spin density plots are shown in Figure 5. It is clear that the location of the unpaired electrons is within the isovalue $= \pm 0.01$. The electrons locations are all the same in the two different edge HGFs and have no relationship with the size or the edge of the graphene flakes. From this calculation, we first found that the spin density of the 555777 divacancy only depends on the vacancy itself. The radical property carbon has more relativities indicating that it is very important to understand the process of the divacancy defect absorption or self-healing reaction.

\section{Conclusions}

In conclusion, we have investigated the geometries and electronic properties of divacancies with two kinds of structures in the different edged hexagonal graphene flakes by the firstprinciples (U) B3LYP/STO-3G and self-consistent-charge 
Energy difference between 585 and 555777 defects
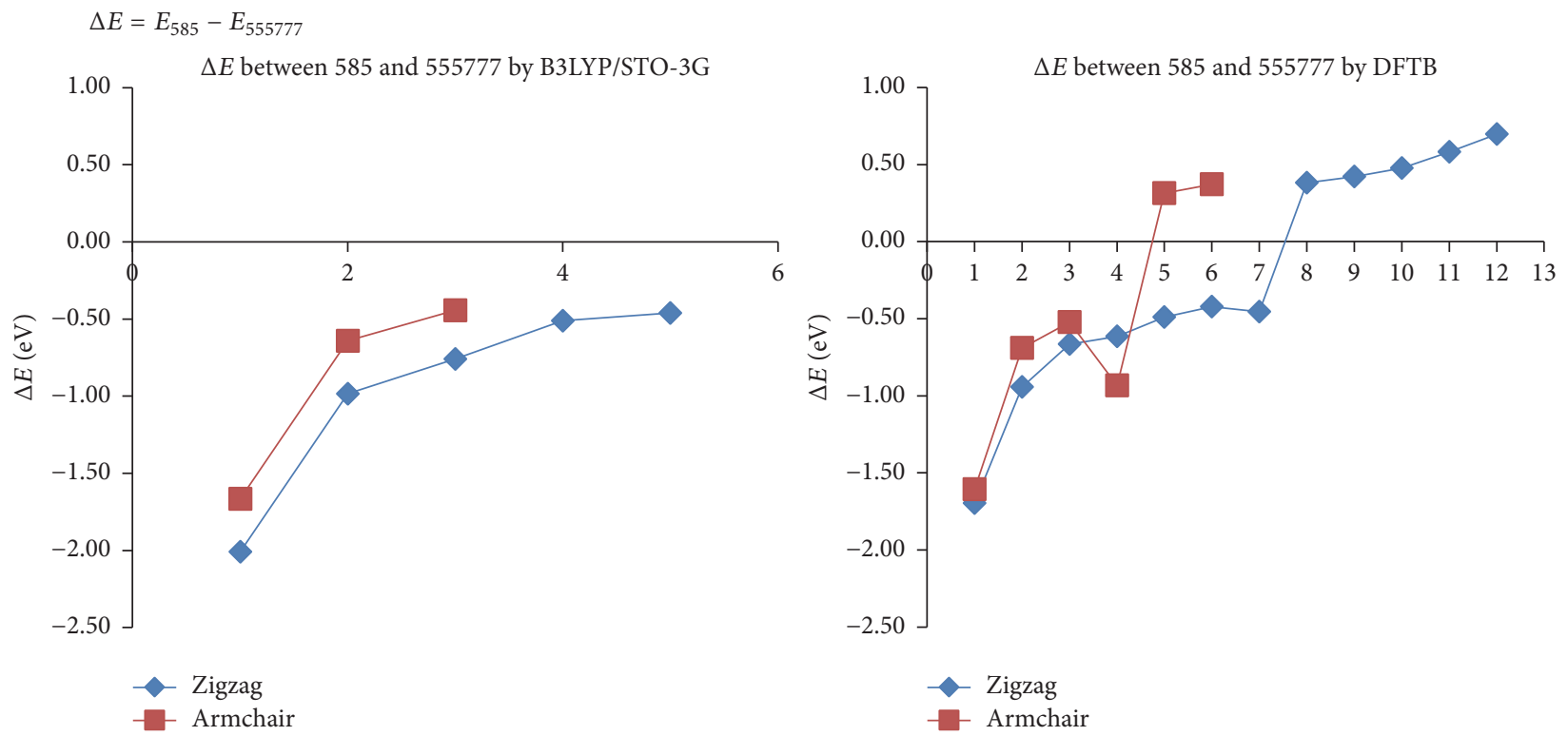

Figure 3: The energy differences between different types of divacancy defects $\left(\Delta E=E_{585}-E_{555777}\right)$ in the same graphene flakes with B3LYP/STO-3G and DFTB methods.
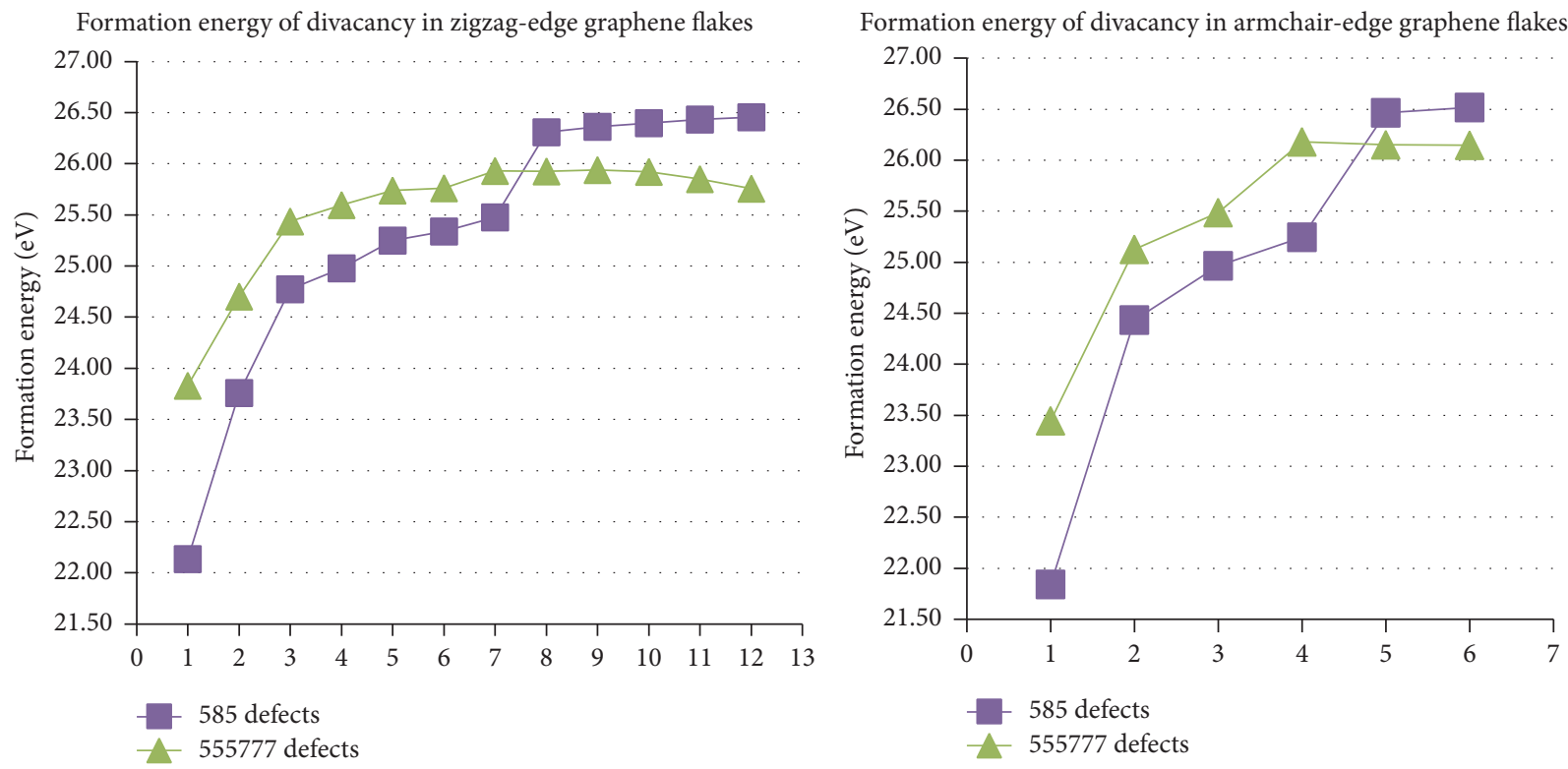

FIGURE 4: The formation energy of the two types of divacancy defects in zigzag- and armchair-edge HGFs by DFTB.

density-functional tight-binding (SCC-DFTB) method. The DFTB reproduced the B3LYP results correspondingly and gave very good results even in the large system, which proved that it is reliable and efficient method in the really large carbon system. It is found that the ground state of the divacancy with 585 configuration is closed shell singlet state and much more stable than the 555777 configurations in the certain small size range, which is preferred to triplet state. More importantly, different from the reported understanding of these properties of divacancy in graphene, we found that the stability of these two kinds of divacancies is highly dependent on the structures themselves and also depends on the HGFs edges characters. Through the investigation of the different states energy comparison and formation energy, we found that the stability of the two divacancies is influenced not only by the size of the graphene flakes but also by the edge type of graphene. In addition, we also calculated the spin density of 555777 and found that the spin density distribution of the 555777 divacancy only depends on the vacancy itself. 


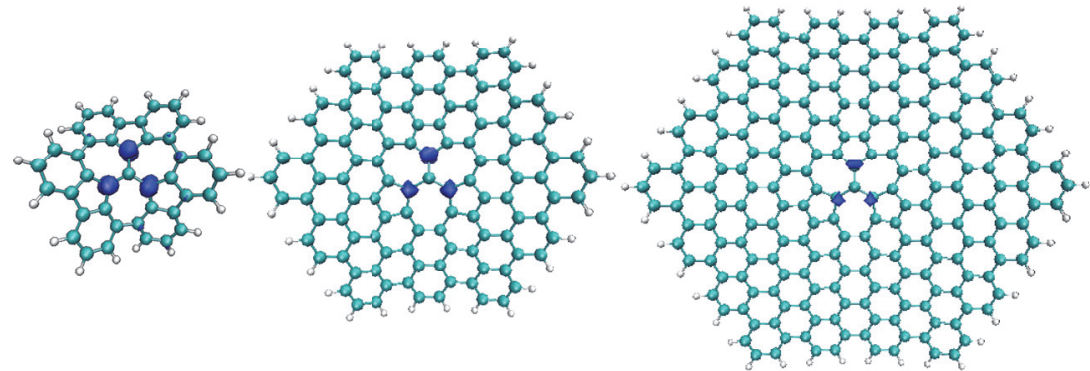

A2

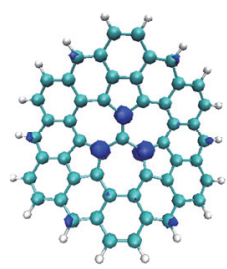

$\mathrm{Z} 2$
A3

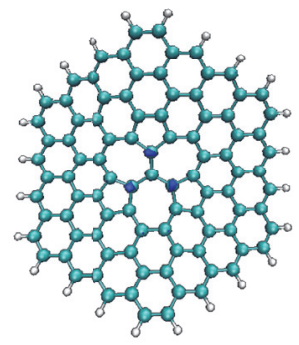

Z3
A4

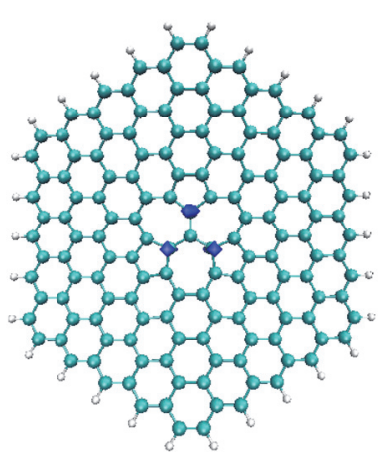

Z4

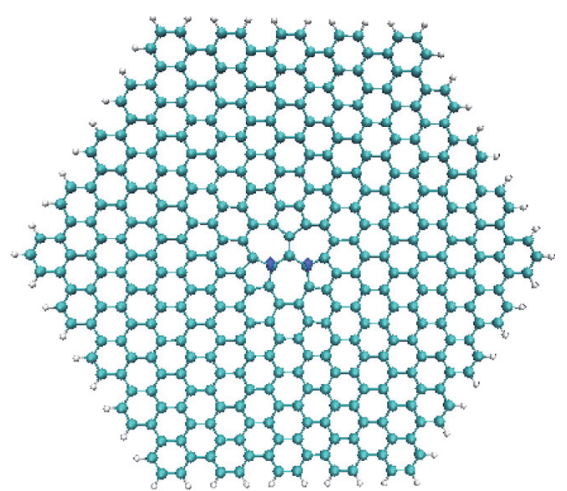

A5

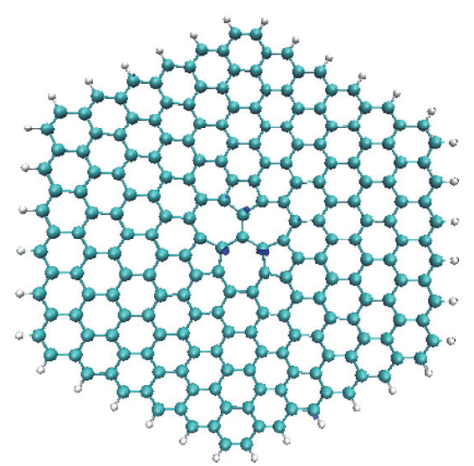

$\mathrm{Z} 5$

FIGURE 5: The spin density distributions of the 555777 divacancy defects in the two type edges of HGFs with the isovalue $= \pm 0.01$.

\section{Competing Interests}

The authors declare that they have no competing interests.

\section{Acknowledgments}

This work was supported by National Natural Science Foundation of China (nos. 21276257, 91534109, and 21503006), the "Strategic Priority Research Program" of the Chinese Academy of Sciences (no. XDA09010103), National Key Projects for Fundamental Research and Development of China (no. 2016YFB0100100), and the Research Foundation for Youth Scholars of Beijing Technology and Business University (no. QNJJ2014-14).

\section{References}

[1] K. S. Novoselov, A. K. Geim, S. V. Morozov et al., "Electric field effect in atomically thin carbon films," Science, vol. 306, no. 5696, pp. 666-669, 2004.

[2] Y. Zhang, H. Zhang, F. Li et al., "Invisible growth of microstructural defects in graphene chemical vapor deposition on copper foil," Carbon, vol. 96, pp. 237-242, 2016.

[3] R. R. Nair, M. Sepioni, I.-L. Tsai et al., "Spin-half paramagnetism in graphene induced by point defects," Nature Physics, vol. 8, no. 3, pp. 199-202, 2012.

[4] J. C. Meyer, C. Kisielowski, R. Erni, M. D. Rossell, M. F. Crommie, and A. Zettl, "Direct imaging of lattice atoms and topological defects in graphene membranes," Nano Letters, vol. 8, no. 11, pp. 3582-3586, 2008.

[5] Q. Fan, J. M. Gottfried, and J. Zhu, "Surface-catalyzed C-C covalent coupling strategies toward the synthesis of low-dimensional carbon-based nanostructures," Accounts of Chemical Research, vol. 48, no. 8, pp. 2484-2494, 2015.

[6] L. Liu, M. Qing, Y. Wang, and S. Chen, "Defects in graphene: generation, healing, and their effects on the properties of graphene: a review," Journal of Materials Science \& Technology, vol. 31, no. 6, pp. 599-606, 2015.

[7] D. W. Boukhvalov and M. I. Katsnelson, "Chemical functionalization of graphene with defects," Nano Letters, vol. 8, no. 12, pp. 4373-4379, 2008.

[8] L. Rodrigo, P. Pou, and R. Pérez, "Graphene monovacancies: electronic and mechanical properties from large scale ab initio simulations," Carbon, vol. 103, pp. 200-208, 2016.

[9] Z. Hou and K. Terakura, "Effect of nitrogen doping on the migration of the carbon adatom and monovacancy in graphene," The Journal of Physical Chemistry C, vol. 119, no. 9, pp. 4922-4933, 2015.

[10] X. Gao, L. Liu, S. Irle, and S. Nagase, "Carbon spiral helix: a nanoarchitecture derived from monovacancy defects in graphene," Angewandte Chemie-International Edition, vol. 49, no. 18, pp. 3200-3202, 2010.

[11] P. Hirunsit, M. Liangruksa, and P. Khanchaitit, "Electronic structures and quantum capacitance of monolayer and multilayer graphenes influenced by $\mathrm{Al}, \mathrm{B}, \mathrm{N}$ and $\mathrm{P}$ doping, and monovacancy: theoretical study," Carbon, vol. 108, pp. 7-20, 2016. 
[12] A. J. Lu and B. C. Pan, "Nature of single vacancy in achiral carbon nanotubes," Physical Review Letters, vol. 92, no. 10, Article ID 105504, 2004.

[13] A. Hashimoto, K. Suenaga, A. Gloter, K. Urita, and S. Iijima, "Direct evidence for atomic defects in graphene layers," Nature, vol. 430, no. 7002, pp. 870-873, 2004.

[14] A. V. Krasheninnikov, P. O. Lehtinen, A. S. Foster, and R. M. Nieminen, "Bending the rules: contrasting vacancy energetics and migration in graphite and carbon nanotubes," Chemical Physics Letters, vol. 418, no. 1-3, pp. 132-136, 2006.

[15] J. Kotakoski, A. V. Krasheninnikov, and K. Nordlund, "Energetics, structure, and long-range interaction of vacancy-type defects in carbon nanotubes: atomistic simulations," Physical Review B, vol. 74, no. 24, Article ID 245420, 2006.

[16] G.-D. Lee, C. Z. Wang, E. Yoon, N.-M. Hwang, D.-Y. Kim, and K. M. Ho, "Diffusion, coalescence, and reconstruction of vacancy defects in graphene layers," Physical Review Letters, vol. 95, no. 20, Article ID 205501, 2005.

[17] R. G. Amorim, A. Fazzio, A. Antonelli, F. D. Novaes, and A. J. R. Da Silva, "Divacancies in graphene and carbon nanotubes," Nano Letters, vol. 7, no. 8, pp. 2459-2462, 2007.

[18] L. Liu, J. Gao, X. Zhang, T. Yan, and F. Ding, "Vacancy inter-layer migration in multi-layered graphene," Nanoscale, vol. 6, no. 11, pp. 5729-5734, 2014.

[19] F. Banhart, J. Kotakoski, and A. V. Krasheninnikov, "Structural defects in graphene," ACS Nano, vol. 5, no. 1, pp. 26-41, 2011.

[20] Y. Kim, J. Ihm, E. Yoon, and G.-D. Lee, "Dynamics and stability of divacancy defects in graphene," Physical Review BCondensed Matter and Materials Physics, vol. 84, no. 7, Article ID 075445, 2011.

[21] Ç. Ö. Girit, J. C. Meyer, R. Erni et al., "Graphene at the edge: stability and dynamics," Science, vol. 323, no. 5922, pp. 17051708, 2009.

[22] D. Porezag, T. Frauenheim, T. Köhler, G. Seifert, and R. Kaschner, "Construction of tight-binding-like potentials on the basis of density-functional theory: application to carbon," Physical Review B, vol. 51, no. 19, pp. 12947-12957, 1995.

[23] M. Elstner, D. Porezag, G. Jungnickel et al., "Self-consistentcharge density-functional tight-binding method for simulations of complex materials properties," Physical Review B, vol. 58, no. 11, pp. 7260-7268, 1998.

[24] M. Elstner, Q. Cui, P. Munih, E. Kaxiras, T. Frauenheim, and M. Karplus, "Modeling zinc in biomolecules with the self consistent charge-density functional tight binding (SCC-DFTB) method: applications to structural and energetic analysis," Journal of Computational Chemistry, vol. 24, no. 5, pp. 565-581, 2003.

[25] M. Elstner, T. Frauenheim, E. Kaxiras, G. Seifert, and S. Suhai, “A self-consistent charge density-functional based tight-binding scheme for large biomolecules," Physica Status Solidi (B) Basic Research, vol. 217, no. 1, pp. 357-376, 2000.

[26] T. Frauenheim, G. Seifert, M. Elstner et al., "Atomistic simulations of complex materials: ground-state and excited-state properties," Journal of Physics Condensed Matter, vol. 14, no. 11, pp. 3015-3047, 2002.

[27] M. J. Frisch, G. W. Trucks, H. B. Schlegel et al., Gaussian 03, Revision C.02, Gaussian Inc, Wallingford, Conn, USA, 2004. 

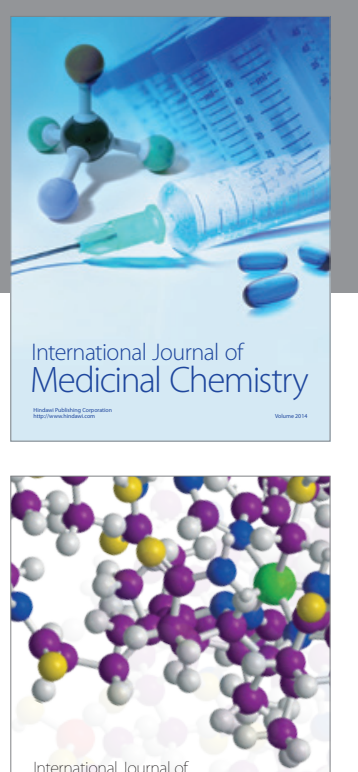

Carbohydrate Chemistry

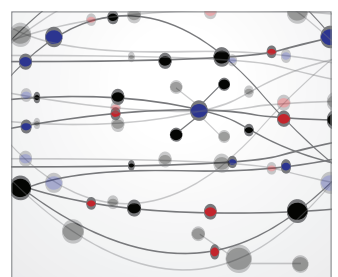

The Scientific World Journal
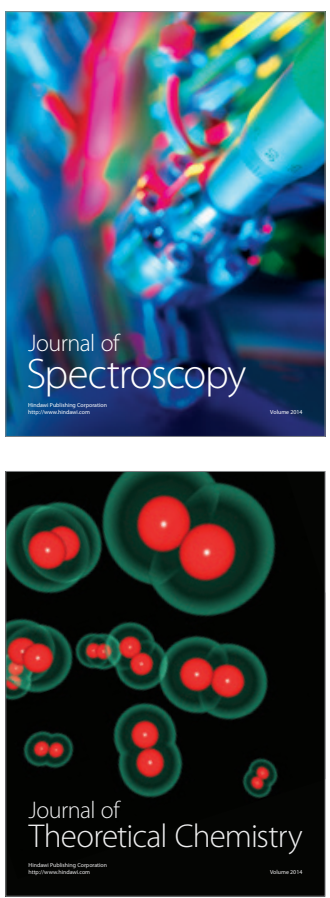
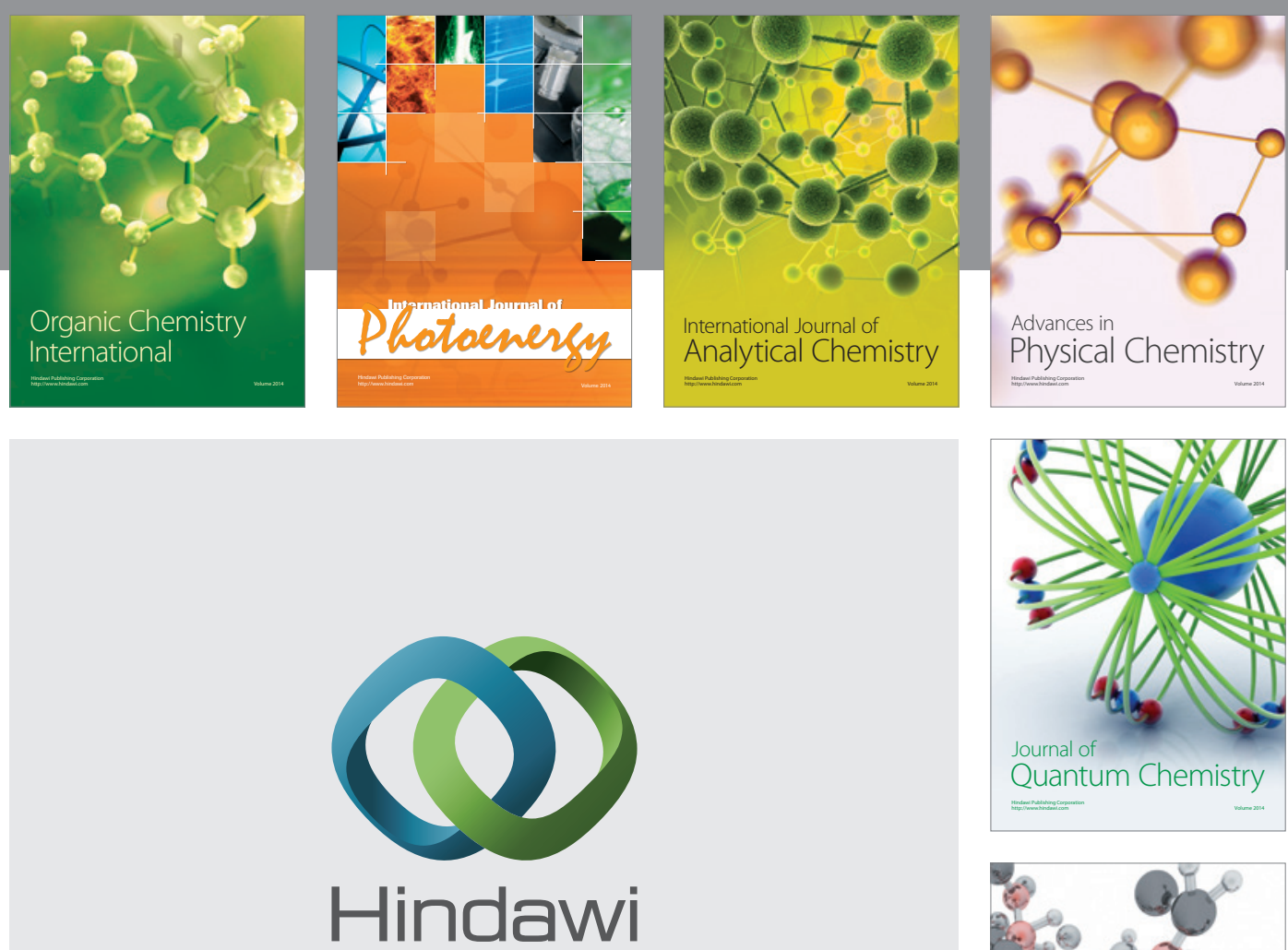

Submit your manuscripts at

https://www.hindawi.com

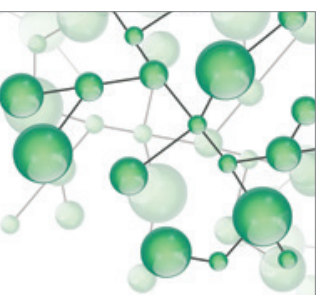

International Journal of

Inorganic Chemistry
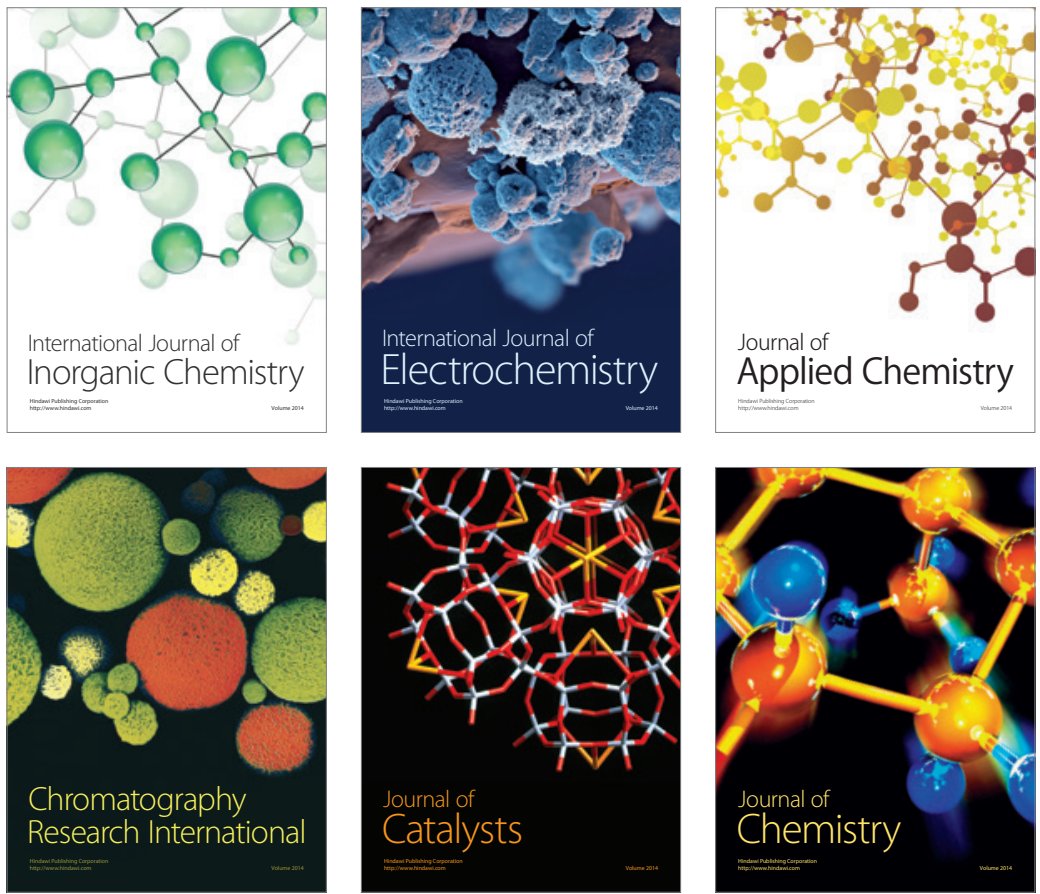

Journal of

Applied Chemistry
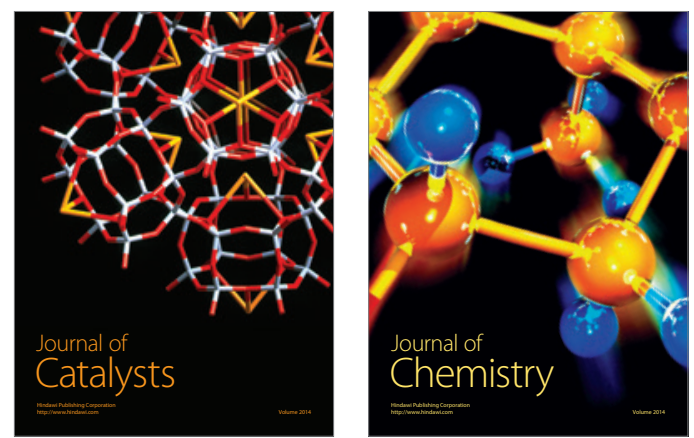
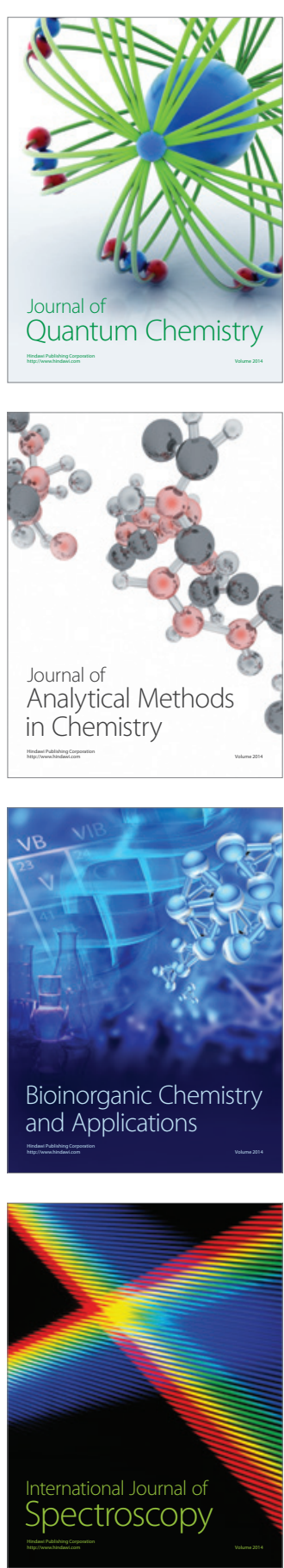